\title{
Are women with type 2 diabetes mellitus more susceptible to cardiovascular complications following coronary angioplasty?: a meta-analysis
}

Pravesh Kumar Bundhun ${ }^{1}$, Manish Pursun ${ }^{2}$ and Feng Huang ${ }^{1 *}$ (])

\begin{abstract}
Background: Scientific reports have shown Type 2 Diabetes Mellitus (T2DM) to be independently associated with adverse outcomes following Percutaneous Coronary Intervention (PCI). However, gender difference has also often been a controversial issue following PCI. Till date, very few meta-analyses have systematically compared the adverse cardiovascular outcomes in male versus female patients with T2DM following PCl. Therefore, we aimed to carry out this analysis in order to find out an answer to this interesting question.

Methods: Electronic databases were searched for English language publications reporting adverse cardiovascular outcomes in male versus female patients with diabetes mellitus respectively following coronary angioplasty. The RevMan 5.3 software was used to analyze selected adverse cardiovascular events whereby Odds Ratios (OR) and 95\% Confidence Intervals (Cl) were the statistical parameters.

Results: A total number of 19,304 patients with T2DM (12,986 male patients versus 6318 female patients) were included in this analysis. At baseline, female patients were older (68.7 versus 62.9 years), with a higher percentage of hypertension (75.6\% versus $66.5 \%$ ) and dyslipidemia (53.3\% versus $50.0 \%$ ) whereas majority of the male patients were smokers (46.3\% versus $14.9 \%$ ). Results of this analysis showed short and long-term mortality to be significantly higher in female patients with T2DM (OR: 1.71, 95\% Cl: 1.46-2.00; $P=0.00001)$, and (OR: 1.20, 95\% Cl: 1.07-1.35; $P=0.002)$ respectively. In addition, women were also more at risk for short and long-term major adverse cardiac events (MACEs) with OR: 1.49, 95\% Cl: 1.07-2.07; $P=0.02$ and OR: $1.15,95 \%$ Cl: 1.04-1.28; $P=0.009$ respectively. Subgroup analysis showed this significant result to have mainly been observed in patients with acute myocardial infarction compared to those with stable coronary artery disease.

Conclusions: Following PCl, women with T2DM were indeed more susceptible to short and long-term cardiovascular complications compared to male patients with the same chronic disease. Even though this result was more applicable to patients with acute myocardial infarction, the fact that women were older with higher co-morbidities at baseline compared to men, should also not be ignored.
\end{abstract}

Keywords: Type 2 diabetes mellitus, Percutaneous coronary intervention, Gender, Female sex, Major adverse cardiac events, Mortality

\footnotetext{
* Correspondence: huangfeng7925@163.com

1 Institute of Cardiovascular Diseases, the First Affiliated Hospital of Guangxi

Medical University, Nanning, Guangxi 530027, People's Republic of China

Full list of author information is available at the end of the article
} 


\section{Background}

With the unhealthy lifestyle adopted by people nowadays, the population of patients with Type 2 Diabetes Mellitus (T2DM) is expected to rise massively in the coming years [1]. Scientific reports have shown T2DM to be independently associated with adverse outcomes following Percutaneous Coronary Intervention (PCI) [2]. However, when gender is to be taken into account, it is still unclear who among men and women, are at higher risks for complications [3, 4].

In a recent meta-analysis dealing with the geographical difference of the interaction of sex with treatment strategy in patients with multi-vessel disease and left main disease, and involving the SYNTAX (Synergy Between PCI With Taxus and Cardiac Surgery), PRECOMBAT (Bypass Surgery Versus Angioplasty Using Sirolimus Eluting Stent in Patients With Left Main Coronary Artery Disease), and BEST (Bypass Surgery and Everolimus Eluting Stent Implantation in the Treatment of Patients With Multivessel Coronary Artery Disease) Randomized Controlled Trials, the authors suggested that the heterogeneity of gendertreatment interaction needed to be well-recognized and considered when deciding the treatment strategy [5].

However, till date, only few meta-analyses have systematically compared adverse cardiovascular outcomes between men and women with T2DM following PCI. Therefore, for a better management of diabetic male and female patients with cardiovascular diseases, we aimed to carry out this analysis in order to find an answer to this interesting question.

\section{Methods}

\section{Searched databases and strategies}

Electronic databases (PubMed, EMBASE and the Cochrane Central Register of Controlled Trials) were searched for English language publications reporting adverse cardiovascular outcomes in male versus female patients with diabetes mellitus following coronary angioplasty.

During this search process, the terms 'diabetes mellitus, gender and percutaneous coronary intervention' were used. The words 'diabetes mellitus' were also replaced by the abbreviation 'T2DM', the term 'gender' was also replaced by the word 'sex, women or men' respectively whereas the words 'percutaneous coronary intervention' were also replaced by the term 'coronary angioplasty' or the abbreviation 'PCI'.

Reference lists of qualified publications were also reviewed for any relevant article. In addition, official websites of major cardiovascular related journals such as the Journal of American College of Cardiology, Journal of Circulation, and Cardiovascular Diabetology were also checked for relevant articles.

\section{Inclusion and exclusion criteria}

Studies were included if they were trials or observational studies comparing adverse clinical outcomes between male and female patients with T2DM following PCI. In addition, studies which dealt with the general population, but consisted of more than $30 \%$ of female patients with T2DM were also qualified for this analysis.

Studies were excluded if:

(a) They were meta-analyses, case studies or letter to editors.

(b) They did not include patients with T2DM.

(c) They did not compare male with female patients.

(d) They did not report adverse cardiovascular outcomes following PCI.

(e) They were duplicates.

\section{Type of participants, outcomes and follow ups}

This analysis included patients with stable coronary artery disease (CAD), acute myocardial infarction (AMI), and specifically patients with STEMI as shown in Table 1.

The outcomes which were analyzed [death, myocardial infarction (MI), repeated revascularization, major adverse cardiac events (MACEs)] have been listed in Table 1.

Death referred to all-cause mortality whereas MACEs consisted mainly of death, MI and revascularization or stroke.

A short-term follow up (less or equal to 30 days) and a longer follow up period (> one year) were considered relevant in this analysis.

\section{Data extraction and review}

The following data were independently extracted by two reviewers (PKB and $\mathrm{MP})$ :

- Names of authors

- Year of publication

- Type of study (observational or randomized trial)

- Number of male patients

- Number of female patients

- Patients' enrollment periods

- Baseline features

- Outcomes which were assessed

- Follow-up periods

- Adverse events which were reported in male versus female patients respectively

Disagreements were resolved by a thorough discussion with the third author $(\mathrm{FH})$.

The PRISMA guideline [6] was followed, and the bias risk was assessed as advised by the Cochrane Collaboration [7], for the only trial which was included.

\section{Statistical analysis}

The RevMan software version 5.3 was used to analyze selected adverse cardiovascular events which were reported between male and female patients with T2DM following 
Table 1 Type of participants, reported outcomes and follow-up periods

\begin{tabular}{llll}
\hline Studies & Outcomes & Follow-up periods & Type of patients \\
\hline Blondal 2012 [9] & MACEs, MI, revascularization, death & 2.1 years & AMl \\
Buja 2012 [26] & MACEs, death, Ml, TVR, TLR & 24.3 months & CAD \\
Holland 2013 [12] & Death, MACEs, revascularization & 5 years & CAD \\
Lin 2013 [27] & Death & 5.4 years & CAD \\
Michelle 2003 [25] & Death & 1 year & CAD \\
Younan 2015 [28] & Death, Ml, TVR, MACEs & 1 year & CAD \\
Epps 2016 [29] & MACEs, death, Ml, revascularization, TLR, TVR & CAD \\
Toyota 2013 [30] & Death, MACEs, TLR, revascularization & In-hospital and 3 years & AMl \\
Cheng 2004 [31] & Death & 30 days & AMl \\
Eitel 2011 [32] & Death & 30 days & STEMl \\
Kosuge 2006 [33] & Death, Ml & In-hospital & AMl \\
Liu 2015 [11] & Death, Ml & 30 days & AMl \\
Qi 2010 [34] & Death, Ml, MACEs, TLR & 30 days & STEMl \\
Roffi 2013 [10] & Death, Ml & 30 days & STEMl \\
Zanchi 2009 [35] & Death & In-hospital & STEMl
\end{tabular}

Abbreviations: MACEs major adverse cardiac events; $M I$ myocardial infarction; TVR target vessel revascularization; $A M I$ acute myocardial infarction; $C A D$ coronary artery disease; STEMI ST segment elevated myocardial infarction

PCI, whereby odds ratios (OR) and 95\% confidence intervals $(\mathrm{CI})$ were used as the statistical parameters.

Heterogeneity [8] across the subgroups analyzing the respective outcomes was assessed using the:

(i) Cochrane $Q$ statistic test $(P \leq 0.05$ was considered statistically significant).

(ii) $\mathrm{I}^{2}$ statistic test (a low value of $\mathrm{I}^{2}$ represented a low heterogeneity. To be more specific, an $\mathrm{I}^{2}$ value below $25 \%$ represented a lower heterogeneity, an $\mathrm{I}^{2}$ value about $50 \%$ represented a moderate heterogeneity whereas an $\mathrm{I}^{2}$ value approaching $100 \%(75-100 \%)$, represented a high heterogeneity).

In this analysis, a fixed $\left(\mathrm{I}^{2}<50 \%\right)$ or a random $\left(\mathrm{I}^{2}>50 \%\right)$ effects model was used depending on the $\mathrm{I}^{2}$ value which was obtained.

Since this analysis involved a low volume of studies, publication bias was visually estimated by assessing funnel plots which were directly generated by the RevMan 5.3 software.

Sensitivity analysis was also carried out (excluding each study one by one and then carrying out a new analysis each time to observe any significant difference in the results).

\section{Ethics and patients' consents}

Ethical approval and patients' consents were not applicable.

\section{Results}

\section{Searched outcomes}

A thorough search from electronic databases ended with a total number of 2695 articles. Following an assessment of the titles and abstracts, 2648 articles were eliminated since they were not linked to this current research. Forty-seven (47) full text articles were assessed for eligibility. However, after a careful assessment of these articles, further publications were eliminated since: they were case studies (3), meta-analysis (1), they did not report adverse clinical outcomes (1), they compared male versus female patients following PCI but, however, they consisted of less than $30 \%$ of women with T2DM (16), they were duplicates (11). Finally, 15 articles were confirmed for this analysis (Fig. 1).

\section{General features of the studies which were included}

Only one randomized trial was included, whereas the other studies were observational studies. This analysis consisted of a total number of 19,304 patients (12,986 male patients and 6318 female patients). Patients' enrollment period ranged from the year 1993 to 2014 (Table 2). Following the bias risk assessment, a grade B was given to the only randomized trial which was included in this analysis.

\section{Baseline features of the male and female patients}

The baseline characteristics of the male and female patients with T2DM have been summarized in Table 3. Male patients had a mean age varying between 56.3 and 66.4 years whereas female patients had a mean age ranging from 58.9 to 74.1 years. Figure 2 shows a graphical representation of age between male and female patients at baseline.

The percentage of female patients suffering from hypertension and dyslipidemia were higher with the exception of one or two studies. On average, a graphical representation of hypertension and dyslipidemia has been shown (Figs. 3 and 4). 


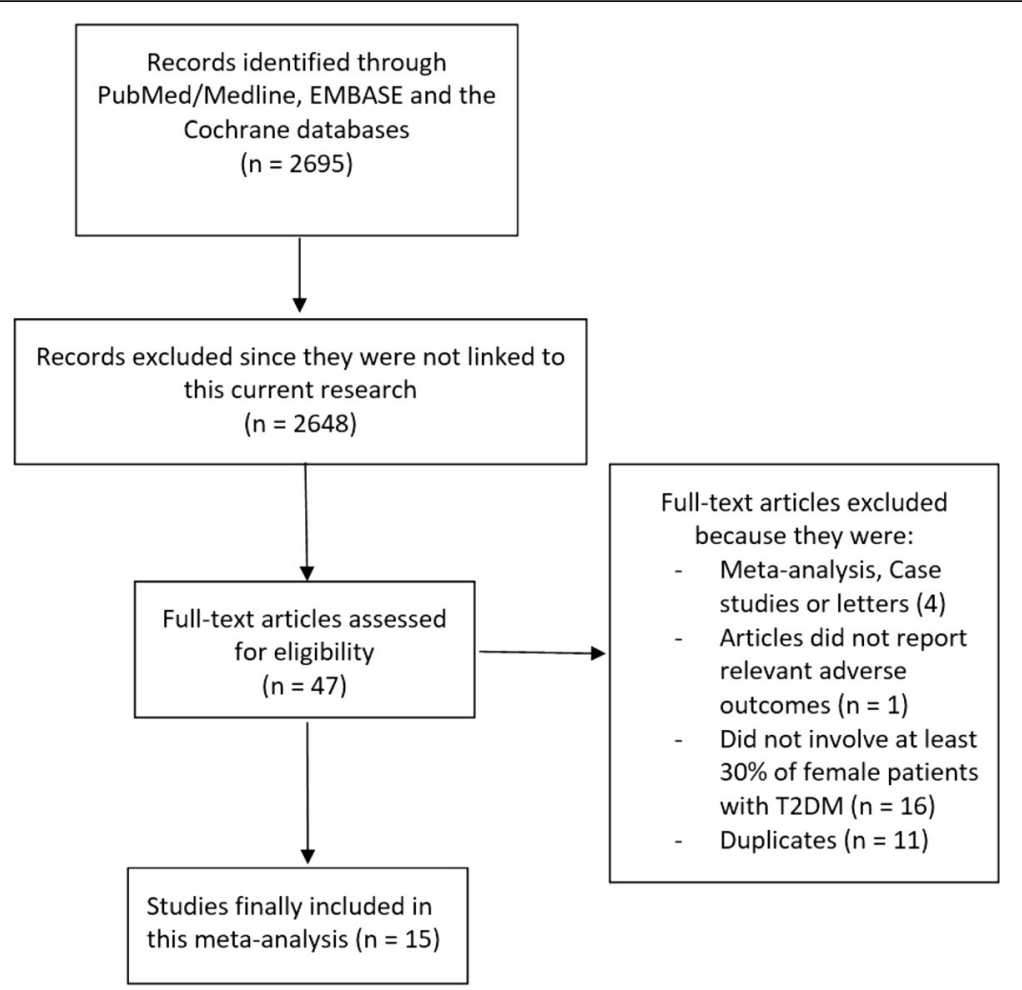

Fig. 1 Flow diagram representing the study selection

Table 2 General features of the studies which were included

\begin{tabular}{lllll}
\hline Studies & Patients' enrollment (years) & No of male patients (n) & No of female patients (n) & Type of study \\
\hline Blondal 2012 [9] & $2006-2009$ & 155 & 142 & Observational \\
Buja 2012 [26] & $2003-2009$ & 1741 & 679 & Observational \\
Holland 2013 [12] & $2001-2005$ & 1666 & 702 & RCT \\
Lin 2013 [27] & $1997-2003$ & 239 & 147 & Observational \\
Michelle 2003 [25] & $1995-1998$ & 2411 & 1074 & Observational \\
Younan 2015 [28] & $2010-2013$ & 100 & 100 & Observational \\
Epps 2016 [29] & $1997-2006$ & 1699 & 1303 & Observational \\
Toyota 2013 [30] & $2005-2007$ & 1046 & 380 & Observational \\
Cheng 2004 [31] & $1993-2000$ & 199 & 61 & Observational \\
Eitel 2011 [32] & - & 56 & 36 & - \\
Kosuge 2006 [33] & $2001-2003$ & 672 & 273 & Observational \\
Liu 2015 [11] & $2010-2014$ & 117 & 52 & Observational \\
Qi 2010 [34] & $2005-2008$ & 348 & 171 & Observational \\
Roffi 2013 [10] & $1997-2010$ & 2412 & 1153 & Observational \\
Zanchi 2009 [35] & $2004-2008$ & 125 & 45 & Observational \\
Total no of patients (n) & & 12,986 & 6318 & \\
\hline Abbrifion & & &
\end{tabular}


Table 3 Baseline features of the patients

\begin{tabular}{|c|c|c|c|c|c|c|}
\hline \multirow[t]{2}{*}{ Studies } & Age (yrs) & $\mathrm{Ht}(\%)$ & Ds (\%) & Cs (\%) & $\mathrm{PCl}^{*}(\%)$ & $C A B G^{*}(\%)$ \\
\hline & $\mathrm{M} / \mathrm{F}$ & $\mathrm{M} / \mathrm{F}$ & $M / F$ & $\mathrm{M} / \mathrm{F}$ & $\mathrm{M} / \mathrm{F}$ & $\mathrm{M} / \mathrm{F}$ \\
\hline Blondal 2012 [9] & $65.0 / 69.3$ & $80.7 / 91.6$ & $62.5 / 69.7$ & $30.3 / 7.00$ & $20.7 / 9.20$ & $3.90 / 3.40$ \\
\hline Buja 2012 [26] & $64.9 / 68.9$ & $73.2 / 82.5$ & $65.9 / 69.5$ & $52.1 / 16.5$ & $11.4 / 12.2$ & - \\
\hline Holland 2013 [12] & $62.2 / 62.9$ & $80.6 / 87.1$ & - & $13.4 / 10.3$ & - & - \\
\hline Lin 2013 [27] & $63.4 / 66.5$ & $74.5 / 82.3$ & $38.5 / 46.9$ & $42.3 / 11.6$ & $53.1 / 47.9$ & $25.1 / 29.9$ \\
\hline Michelle 2003 [25] & $63.6 / 65.7$ & 61.8/73.6 & $46.8 / 44.5$ & - & $12.6 / 12.1$ & $10.5 / 7.00$ \\
\hline Younan 2015 [28] & $57.9 / 58.9$ & $64.0 / 56.0$ & $62.0 / 60.0$ & $45.0 / 3.00$ & $22.0 / 18.0$ & $2.00 / 2.00$ \\
\hline Epps 2016 [29] & $65.0 / 69.0$ & $69.4 / 79.0$ & $70.4 / 70.4$ & $21.7 / 19.1$ & $33.8 / 28.7$ & $22.0 / 15.4$ \\
\hline Toyota 2013 [30] & $64.5 / 74.1$ & $76.7 / 81.0$ & - & $51.9 / 14.6$ & - & - \\
\hline Cheng 2004 [31] & $61.0 / 67.0$ & $45.0 / 67.1$ & $42.4 / 40.8$ & $65.2 / 5.1$ & - & $0.0 / 0.0$ \\
\hline Eitel 2011 [32] & $64.0 / 72.0$ & $68.0 / 76.0$ & $33.0 / 30.0$ & $43.0 / 34.0$ & - & - \\
\hline Kosuge 2006 [33] & $65.0 / 73.0$ & $52.0 / 64.0$ & $33.0 / 37.0$ & $57.0 / 17.0$ & - & - \\
\hline Liu 2015 [11] & $56.3 / 69.5$ & $74.4 / 71.2$ & $45.3 / 69.2$ & $73.5 / 13.5$ & $22.2 / 7.70$ & - \\
\hline Qi 2010 [34] & 63.9/71.7 & $51.0 / 68.8$ & $39.6 / 44.9$ & $65.6 / 9.80$ & $5.50 / 4.10$ & - \\
\hline Roffi 2013 [10] & $66.4 / 73.0$ & 70.7/79.4 & $62.8 / 61.7$ & $36.3 / 19.7$ & - & - \\
\hline Zanchi 2009 [35] & $60.3 / 67.3$ & $55.7 / 75.0$ & $47.5 / 48.4$ & $51.4 / 27.4$ & - & $5.20 / 2.40$ \\
\hline
\end{tabular}

Abbreviations: yrs. years; $H t$ hypertension; $D s$ dyslipidemia; Cs current smoker; $P C l$ percutaneous coronary intervention; $C A B G$ coronary artery bypass surgery; $M$ males; $F$ females

${ }^{*}$ signified previous $\mathrm{PCl}$ or previous CABG

At baseline, majority of male patients were smokers, compared to female patients as shown in Fig. 5. To summarize the baseline features, female patients were older, and had a higher risk for co-morbidities whereas male patients were heavy smokers.

The medication use at baseline (if reported) has also been summarized in Table 4 .

\section{Short-term adverse outcomes observed between men versus women with T2DM}

The main results have been summarized in Table 5 .
Results of this analysis showed that during a short-term follow up period, mortality and MACEs were significantly higher in women with T2DM (OR: 1.71, 95\% CI: $1.46-$ $2.00 ; P=0.00001, \mathrm{I}^{2}=0 \%$ ), and (OR: $1.49,95 \%$ CI: $1.07-$ 2.07; $\left.P=0.02, \mathrm{I}^{2}=37 \%\right)$ respectively as shown in Fig. 6 .

However, even if short-term MI was also higher in these women (OR: $1.26,95 \% \mathrm{CI}: 0.77-2.07 ; P=0.36, \mathrm{I}^{2}=63 \%$ ), the result was not statistically significant as shown in Fig. 7 .

Another subgroup analysis was carried out only with STEMI patients. Mortality and MI were significantly higher with women following PCI with OR: 1.67, 95\% CI:

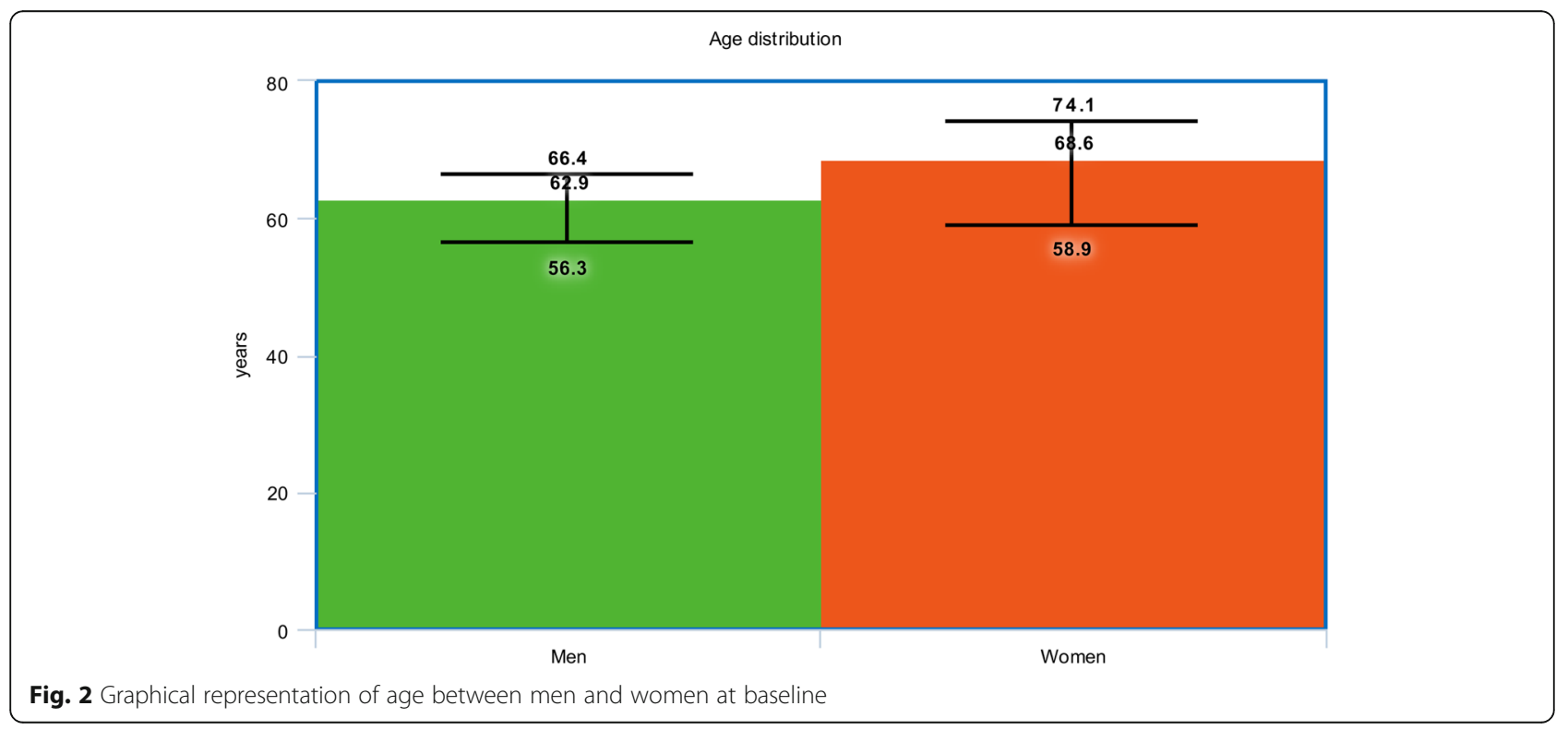




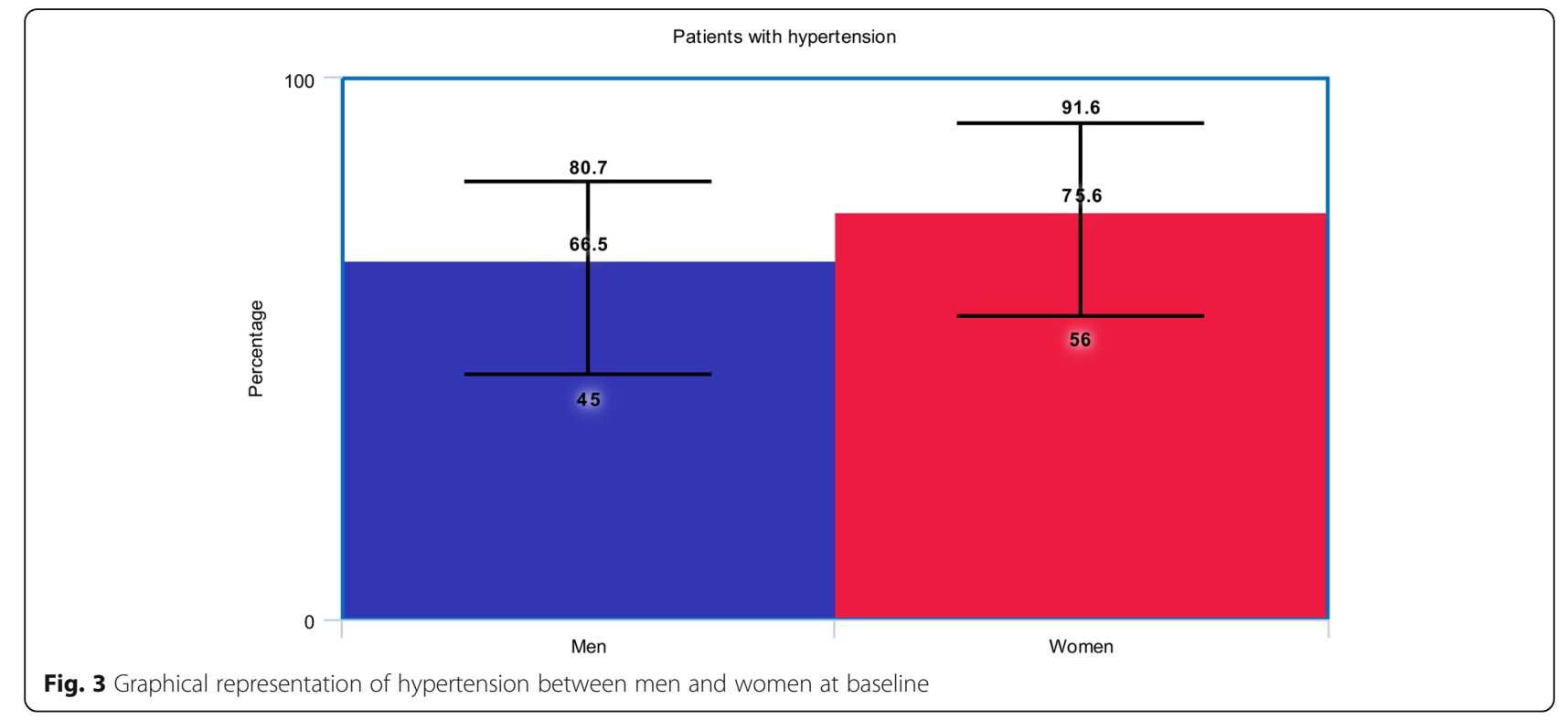

1.37-2.02; $P=0.00001, \mathrm{I}^{2}=0 \%$ and OR: $1.61,95 \% \mathrm{CI}$ : $1.15-2.25 ; P=0.006, \mathrm{I}^{2}=0 \%$ respectively (Fig. 8).

\section{Long-term adverse outcomes observed between men versus women with T2DM}

When the adverse outcomes were analyzed during a longer follow-up period, mortality and MACEs were still significantly higher in female patients with T2DM, with OR: 1.20, 95\% CI: $1.07-1.35 ; P=0.002$ and OR: 1.15 , 95\% CI: $1.04-$ 1.28; $P=0.009$ respectively (Fig. 9). However, even if MI was also higher in female patients with OR: $1.20,95 \% \mathrm{CI}$ : $0.95-1.53 ; P=0.13$, the result was not significant (Fig. 9).
Long-term repeated revascularization was similarly manifested between male and female patients with T2DM, with OR: $0.93,95 \%$ CI: $0.76-1.13 ; P=0.46$ as shown in Fig. 10.

Another subgroup analysis was carried out only including diabetic patients with stable coronary artery disease. Even if a higher mortality, MI, MACEs and repeated revascularization were observed in women, with OR: $1.12,95 \%$ CI: $0.99-1.28 ; P=0.07, \mathrm{I}^{2}=3 \%$, OR: 1.24, 95\% CI: $0.96-1.60 ; P=0.10, \mathrm{I}^{2}=0.10$, OR: 1.10 , 95\% CI: $0.98-1.24 ; P=0.12, \mathrm{I}^{2}=0 \%$ and OR: $1.07,95 \%$ CI: $0.94-1.21 ; P=0.33, \mathrm{I}^{2}=0 \%$ respectively, the results were not statistically significant as shown in Fig. 11.

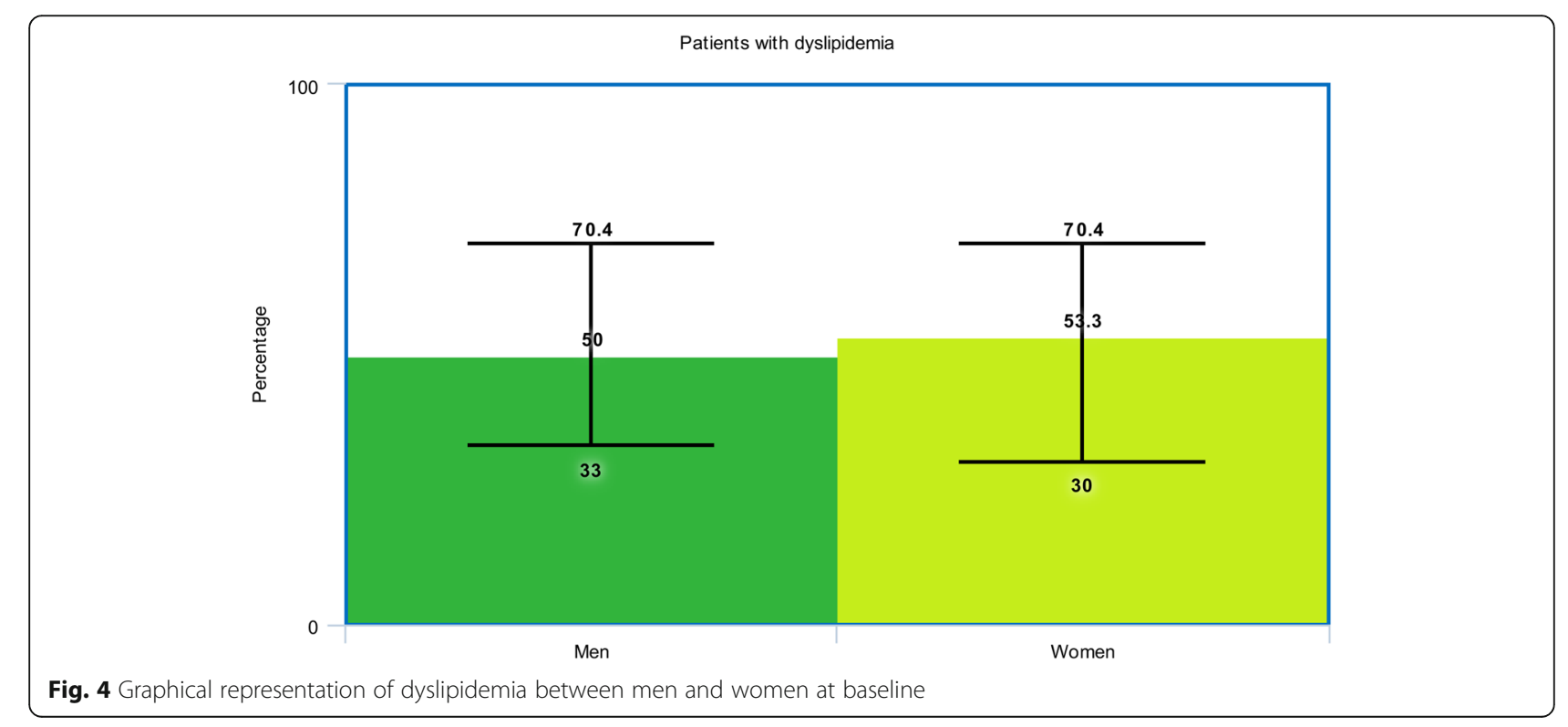


Cigarette smokers

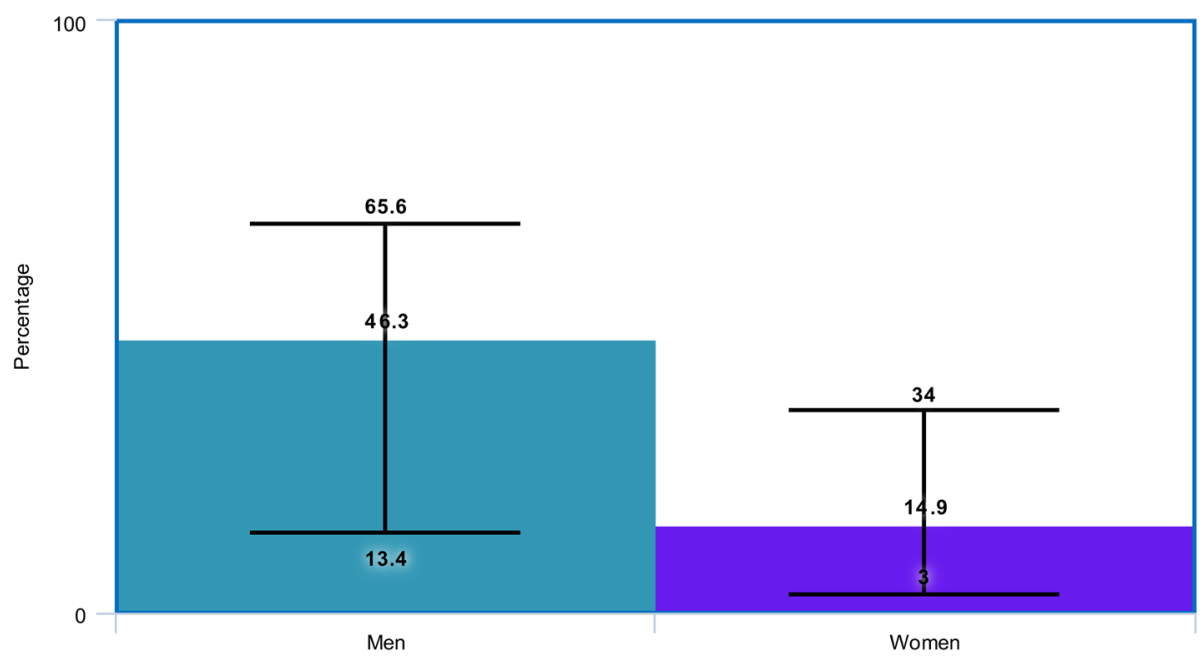

Fig. 5 Graphical representation of smokers between men and women at baseline

\section{Sensitivity analysis}

Sensitivity analyses were carried out by excluding each study one by one, and carrying out a new analysis. Sensitivity analysis which was carried out on the subgroup assessing for short-term mortality did not show any deviation from the main result of this analysis. However, for the subgroup analyzing MI, excluding study Epps2016 showed a significantly higher MI in women with T2DM, with OR: $1.56,95 \%$ CI: $1.15-2.11 ; P=0.005, \mathrm{I}^{2}=44 \%$.

Sensitivity analysis which was carried out on the subgroup assessing for long-term mortality did not show any significant difference compared to the main result. However, in the subgroup assessing for MACEs, excluding study Holland2013 and Toyota2013 respectively, showed a non-significant result with OR: 1.12, 95\% CI: 0.99-1.27; $P=0.08$ and OR: $1.10,95 \%$ CI: $0.98-1.23 ; P=0.11$ respectively.

\section{Publication bias}

Based on a visual assessment of the funnel plots which were obtained (Figs. 12 and 13), there has been little evidence of publication bias across the studies that assessed

Table 4 Medication profile at baseline

\begin{tabular}{|c|c|c|c|c|c|}
\hline \multirow[t]{2}{*}{ Studies } & Insulin therapy & Aspirin & Clopidogrel & Statin & Beta-blocker \\
\hline & Male/female & Male/female & Male/female & Male/female & Male/female \\
\hline Blondal 2012 [9] & - & $99.4 / 96.5$ & $96.8 / 90.9$ & $76.8 / 73.9$ & $82.6 / 76.8$ \\
\hline Buja 2012 [26] & $30.6 / 40.7$ & - & - & - & - \\
\hline Holland 2013 [12] & - & $91.0 / 88.0$ & $91.0 / 88.0$ & $76.0 / 73.0$ & $72.0 / 75.0$ \\
\hline Lin 2013 [27] & - & - & - & $17.6 / 19.7$ & $43.5 / 40.1$ \\
\hline Michelle 2003 [25] & - & - & - & - & - \\
\hline Younan 2015 [28] & $72.0 / 68.0$ & - & - & $41.0 / 39.0$ & $34.0 / 38.0$ \\
\hline Epps 2016 [29] & - & $96.0 / 94.4$ & - & $68.5 / 65.9$ & 77.0/75.6 \\
\hline Toyota 2013 [30] & $4.10 / 4.70$ & $12.6 / 13.5$ & $7.00 / 13.0$ & $11.4 / 18.4$ & - \\
\hline Cheng 2004 [31] & - & - & - & - & - \\
\hline Eitel 2011 [32] & - & $100 / 98.0$ & $100 / 98.0$ & 98.0/99.0 & $99.0 / 97.0$ \\
\hline Kosuge 2006 [33] & - & - & - & - & - \\
\hline Liu 2015 [11] & - & - & - & - & - \\
\hline Qi 2010 [34] & - & $100 / 100$ & $100 / 100$ & $91.7 / 91.2$ & $71.6 / 68.6$ \\
\hline Roffi 2013 [10] & - & - & - & - & - \\
\hline Zanchi 2009 [35] & - & - & - & - & - \\
\hline
\end{tabular}


Table 5 Results of this analysis

\begin{tabular}{|c|c|c|c|c|}
\hline Outcomes analyzed & No of studies involved (n) & OR with $95 \% \mathrm{Cl}$ & $P$ value & $1^{2}(\%)$ \\
\hline \multicolumn{5}{|l|}{ Short term outcomes } \\
\hline Mortality & 9 & $1.71[1.46-2.00]$ & 0.00001 & 0 \\
\hline $\mathrm{Ml}$ & 5 & $1.26[0.77-2.07]$ & 0.36 & 63 \\
\hline MACEs & 2 & $1.49[1.07-2.07]$ & 0.02 & 37 \\
\hline \multicolumn{5}{|l|}{ Long-term outcomes } \\
\hline Mortality & 8 & $1.20[1.07-1.35]$ & 0.002 & 41 \\
\hline $\mathrm{Ml}$ & 4 & $1.20[0.95-1.53]$ & 0.13 & 0 \\
\hline MACES & 6 & $1.15[1.04-1.28]$ & 0.009 & 43 \\
\hline RR & 6 & $0.93[0.76-1.13]$ & 0.46 & 56 \\
\hline
\end{tabular}

Abbreviations: $M I$ myocardial infarction; $M A C E s$ major adverse cardiac events; $O R$ odds ratios; $C l$ confidence intervals; $R R$ repeated revascularization

all of these cardiovascular outcomes observed between male and female patients with T2DM following PCI.

\section{Discussion}

A worse prognosis is often associated with patients suffering from T2DM following coronary angioplasty compared to patients without T2DM. However, the fact that whether women or men with diabetes mellitus are at higher risk of suffering from cardiovascular complications is still controversial. Therefore, in this analysis, we aimed to systematically compare the adverse outcomes observed between male and female patients with T2DM following PCI.
The current results showed significantly higher short and long-term mortality and MACEs in female patients with T2DM. This result was more prominent among diabetic patients with acute myocardial infarction. Such a result could have been due to the fact that an increased percentage of co-morbidities such as hypertension and dyslipidemia was observed in female patients with reference to this current analysis. For example, study Blondal2012 [9] consisted of $80.2 \%$ versus $91.6 \%$ of male and female patients with hypertension respectively, and $62.5 \%$ versus $69.7 \%$ of male and female patients with dyslipidemia respectively. Other registries also showed women to suffer more comorbidities than men. In addition, female patients were

\begin{tabular}{|c|c|c|c|c|c|c|c|c|c|}
\hline \multirow{3}{*}{$\begin{array}{l}\text { Study or Subgroup } \\
1.3 .1 \text { Mortality }\end{array}$} & \multicolumn{2}{|c|}{ Diabetic Women } & \multicolumn{2}{|c|}{ Diabetic Men } & \multirow[b]{2}{*}{ Weight } & \multirow{2}{*}{$\begin{array}{c}\text { Odds Ratio } \\
\text { M-H, Fixed, } 95 \% \mathrm{Cl}\end{array}$} & & \multirow{2}{*}{$\begin{array}{c}\text { Odds Ratio } \\
\text { M-H, Fixed, } 95 \% \mathrm{Cl}\end{array}$} \\
\hline & \multirow[t]{2}{*}{ Events } & \multirow[t]{2}{*}{ Total } & \multirow[t]{2}{*}{ Events } & \multirow[t]{2}{*}{ Total } & & & & & \\
\hline & & & & & Weight & & & & \\
\hline Cheng2004 & 9 & 61 & 15 & 199 & $2.2 \%$ & $2.12[0.88,5.13]$ & & & \\
\hline Eitel2011 & 4 & 36 & 3 & 56 & $0.8 \%$ & $2.21[0.46,10.51]$ & & & \\
\hline Epps2016 & 20 & 1303 & 22 & 1699 & $6.9 \%$ & $1.19[0.65,2.19]$ & & & \\
\hline Kosuge2006 & 26 & 273 & 35 & 672 & $6.7 \%$ & $1.92[1.13,3.25]$ & & & \\
\hline Liu2015 & 5 & 52 & 1 & 117 & $0.2 \%$ & $12.34[1.40,108.47]$ & & & \\
\hline Qi2010 & 10 & 171 & 20 & 348 & $4.5 \%$ & $1.02[0.47,2.23]$ & & & \\
\hline Roffi2013 & 186 & 1153 & 245 & 2412 & $48.7 \%$ & $1.70[1.39,2.09]$ & & 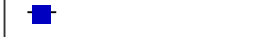 & \\
\hline Toyota2013 & 33 & 380 & 51 & 1046 & $9.1 \%$ & $1.86[1.18,2.92]$ & & & \\
\hline $\begin{array}{l}\text { Zanchi2009 } \\
\text { Subtotal }(95 \% \mathrm{Cl})\end{array}$ & 5 & $\begin{array}{r}45 \\
3474\end{array}$ & 6 & $\begin{array}{r}125 \\
6674\end{array}$ & $\begin{array}{r}1.0 \% \\
80.2 \%\end{array}$ & $\begin{array}{l}2.48[0.72,8.56] \\
1.71[1.46,2.00]\end{array}$ & & & \\
\hline Total events & 298 & & 398 & & & & & & \\
\hline $\begin{array}{l}\text { Heterogeneity: } \mathrm{Chi}^{2}= \\
\text { Test for overall effect: }\end{array}$ & $\begin{array}{l}.21, d f=8( \\
Z=6.58(P\end{array}$ & $\begin{array}{l}=0.51) ; \\
0.00001)\end{array}$ & $I^{2}=0 \%$ & & & & & & \\
\hline 1.3.2 Major Adverse & Sardiac Eve & & & & & & & & \\
\hline Qi2010 & 18 & 171 & 34 & 348 & $7.3 \%$ & $1.09[0.59,1.99]$ & & & \\
\hline $\begin{array}{l}\text { Toyota2013 } \\
\text { Subtotal }(95 \% \mathrm{Cl})\end{array}$ & 43 & $\begin{array}{l}380 \\
551\end{array}$ & 72 & $\begin{array}{l}1046 \\
1394\end{array}$ & $\begin{array}{l}12.5 \% \\
19.8 \%\end{array}$ & $\begin{array}{l}1.73[1.16,2.57] \\
1.49[1.07,2.07]\end{array}$ & & & \\
\hline Total events & 61 & & 106 & & & & & & \\
\hline Heterogeneity: $\mathrm{Chi}^{2}=$ & $.58, \mathrm{df}=1$ & $=0.21$; & $1^{2}=37 \%$ & & & & & & \\
\hline Test for overall effect: & $Z=2.35(P=$ & $0.02)$ & & & & & & & \\
\hline Total $(95 \% \mathrm{Cl})$ & & 4025 & & 8068 & $100.0 \%$ & $1.66[1.44,1.92]$ & & $\gamma$ & \\
\hline Total events & 359 & & 504 & & & & & & \\
\hline $\begin{array}{l}\text { Heterogeneity: } \mathrm{Chi}^{2}= \\
\text { Test for overall effect: } \\
\text { Test for subgroup diffe }\end{array}$ & $\begin{array}{l}.25, \mathrm{df}=10 \\
Z=6.95(P \\
\text { rences: } \mathrm{Chi}^{2}\end{array}$ & $\begin{array}{l}=0.51) \\
.00001) \\
0.53, \mathrm{df}\end{array}$ & $\begin{array}{l}; l^{2}=0 \% \\
f=1(P=\end{array}$ & $0.47), 1^{2}$ & $=0 \%$ & 0.01 & $\begin{array}{cc}0.1 & 1 \\
\text { Favours [diabetic women] }\end{array}$ & 1 Favours [diabetic men] & 100 \\
\hline g. 6 Short-term mo & ality and & Es ob & bserved & twee & n womer & $\mathrm{n}$ versus men with T2DM & I following $\mathrm{PCl}$ & & \\
\hline
\end{tabular}




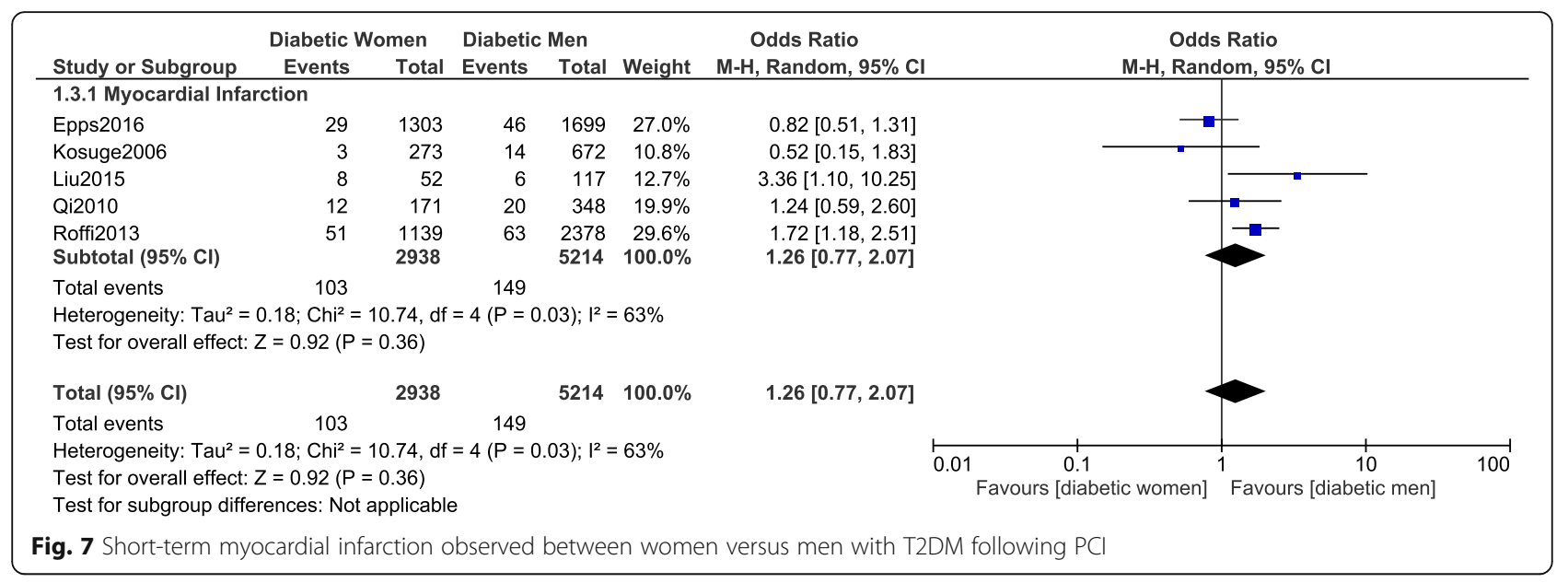

also older when compared to the male population and this observation was clearly visible at baseline.

Similarly, Insights from a nationwide registry (19972010) showed significantly higher in-hospital mortality among women with diabetes when compared to male patients with the same disease following PCI [10]. In addition, at baseline, female patients were older (73.0 versus 66.4 years), majority with hypertension compared to male patients at baseline.

Recently, Liu et al. also showed higher complications to be associated with women (with diabetes mellitus) when compared to men, following coronary angioplasty [11]. In their study, women were older, majority with dyslipidemia compared to men. The Bypass Angioplasty Revascularization Investigation (BARI) 2 Diabetes Trial also supported the result of this current analysis suggesting that higher co-morbidities increased severity of symptoms which were present in these women with T2DM [12]. It should be noted that these women with T2DM also had an advanced age and a longer duration of diabetes mellitus when compared to male patients from the BARI 2 Diabetes Trial. To support the fact that higher co-morbidities which were associated with women might have been responsible for such outcomes, a patient-level pooled analysis of randomized controlled trials showed that among women who underwent PCI with drug eluting stents (DES), chronic kidney disease was associated with a main risk for MACE [13].

Kautzky-Willer et al. showed that women with T2DM had a worse diabetic profile and could achieve therapeutic goals less frequently compared to males with T2DM and suggested more aggressive treatments in women [14]. Moreover, even if Champney et al. concluded that diabetes mellitus was no longer a main risk

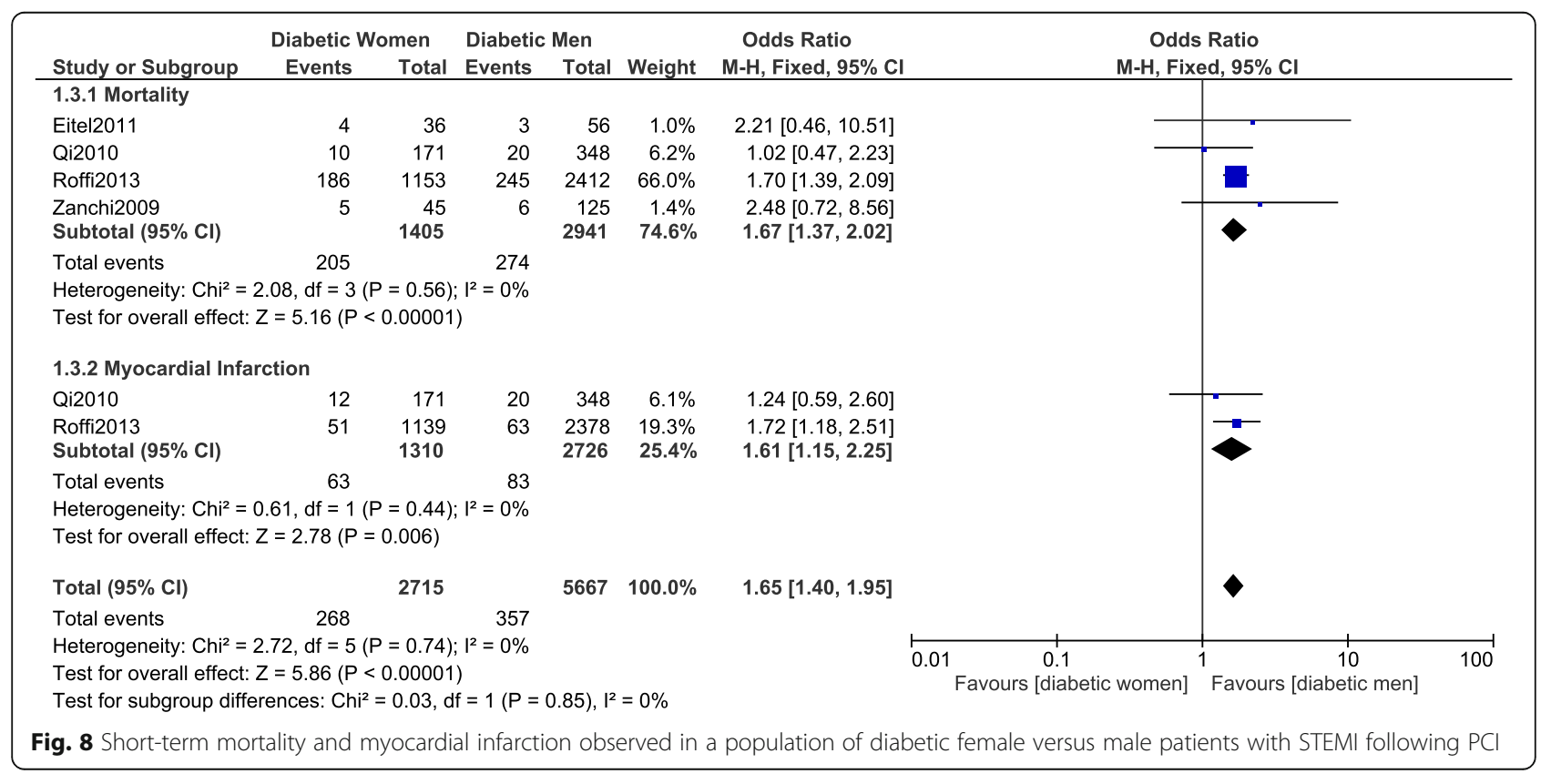




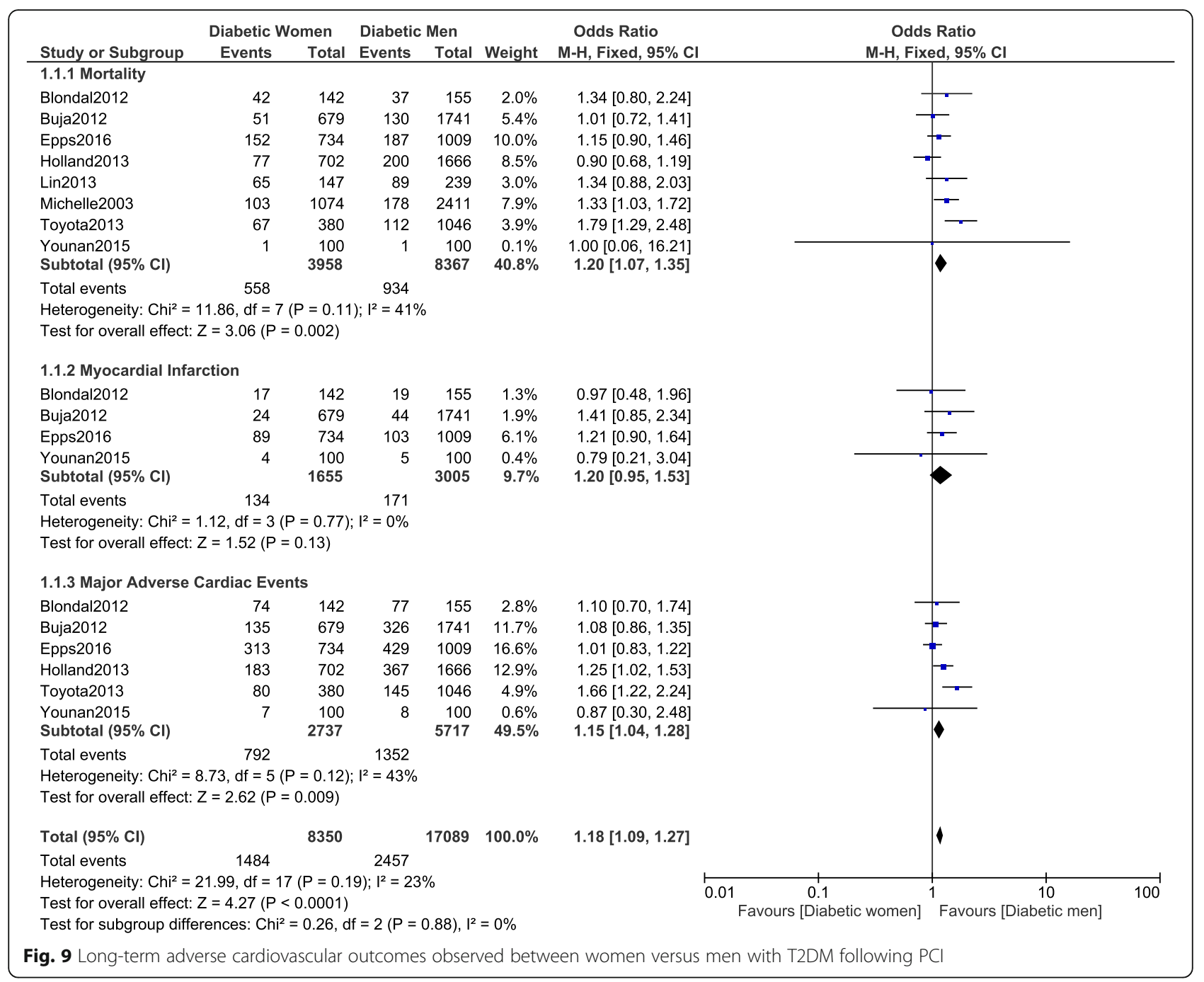

\begin{tabular}{|c|c|c|c|c|c|c|c|c|c|}
\hline \multirow[b]{2}{*}{ Study or Subgroup } & \multicolumn{2}{|c|}{ Diabetic Women } & \multicolumn{2}{|c|}{ Diabetic Men } & \multirow[b]{2}{*}{ Weight } & \multirow{2}{*}{$\begin{array}{l}\text { Odds Ratio } \\
\text { M-H, Random, } 95 \% \mathrm{Cl}\end{array}$} & \multirow{2}{*}{\multicolumn{3}{|c|}{$\begin{array}{c}\text { Odds Ratio } \\
\text { M-H, Random, } 95 \% \mathrm{Cl}\end{array}$}} \\
\hline & Events & Total & Events & Total & & & & & \\
\hline \multicolumn{10}{|c|}{ 1.1.1 Repeated revascularization } \\
\hline Blondal2012 & 18 & 142 & 29 & 155 & $7.4 \%$ & $0.63[0.33,1.19]$ & & & \\
\hline Buja2012 & 96 & 679 & 263 & 1741 & $22.0 \%$ & $0.93[0.72,1.19]$ & & & \\
\hline Epps2016 & 127 & 734 & 164 & 1009 & $21.9 \%$ & $1.08[0.84,1.39]$ & & - & \\
\hline Holland2013 & 246 & 702 & 533 & 1666 & $26.4 \%$ & $1.15[0.95,1.38]$ & & - & \\
\hline Toyota2013 & 111 & 380 & 384 & 1046 & $21.9 \%$ & $0.71[0.55,0.92]$ & - & & \\
\hline $\begin{array}{l}\text { Younan2015 } \\
\text { Subtotal }(95 \% \mathrm{Cl})\end{array}$ & 1 & $\begin{array}{r}100 \\
2737\end{array}$ & 1 & $\begin{array}{r}100 \\
5717\end{array}$ & $\begin{array}{r}0.5 \% \\
100.0 \%\end{array}$ & $\begin{array}{c}1.00[0.06,16.21] \\
0.93[0.76,1.13]\end{array}$ & & & \\
\hline Total events & 599 & & 1374 & & & & & & \\
\hline \multicolumn{10}{|c|}{$\begin{array}{l}\text { Heterogeneity: } \mathrm{Tau}^{2}=0.03 ; \mathrm{Chi}^{2}=11.36, \mathrm{df}=5(P=0.04) ; \mathrm{I}^{2}=56 \% \\
\text { Test for overall effect: } Z=0.73(P=0.46)\end{array}$} \\
\hline Total $(95 \% \mathrm{Cl})$ & & 2737 & & 5717 & $100.0 \%$ & $0.93[0.76,1.13]$ & & & \\
\hline Total events & 599 & & 1374 & & & & & & \\
\hline $\begin{array}{l}\text { Heterogeneity: } \mathrm{Tau}^{2}= \\
\text { Test for overall effect: } \\
\text { Test for subgroup diffe }\end{array}$ & $\begin{array}{l}0.03 ; \mathrm{Chi}^{2}= \\
Z=0.73(\mathrm{P}= \\
\text { rences: Not }\end{array}$ & $\begin{array}{l}1.36, \mathrm{df}= \\
0.46) \\
\text { pplicable }\end{array}$ & $=5(P=0$ & $.04) ;\left.\right|^{2}=$ & $=56 \%$ & & 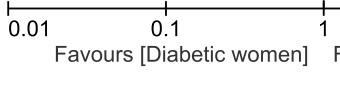 & $\begin{array}{c}10 \\
\text { Favours [Diabetic men] }\end{array}$ & 100 \\
\hline
\end{tabular}




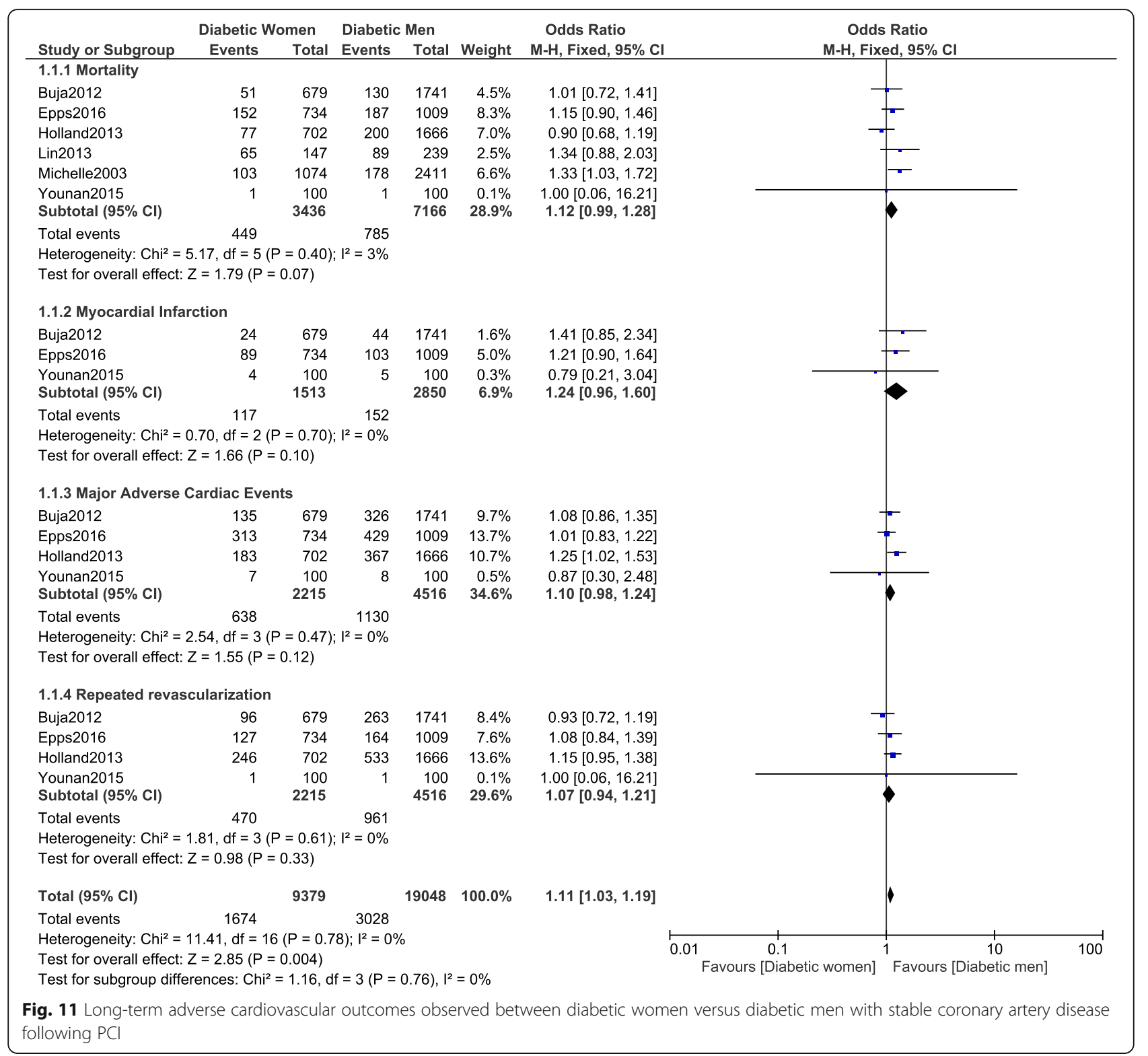

factor for women who underwent coronary angioplasty, their results showed diabetes mellitus to have a strong association with adverse cardiovascular outcomes in women compared to men when gender and diabetes were to be considered [15].

A meta-analysis of sex-related differences in outcomes following primary PCI for ST segment elevation MI also showed female gender to be associated with significantly higher short-term mortality with OR: 1.10, 95\% CI: $1.02-$ 1.18; $P=0.02, \mathrm{I}^{2}=83 \%$ [16]. However, the result was highly heterogeneous. It should be noted that the analysis consisted of 5832 women with T2DM which was almost comparable to this current analysis. In addition, no significant difference in mid-term mortality was observed with OR: $1.01,95 \%$ CI: 0.99-1.03; $P=0.46, \mathrm{I}^{2}=1 \%$.
The 'smoking paradox' [17] is another possible reason which might have contributed to a low level of adverse clinical outcomes in these male patients with T2DM following PCI. At baseline, majority of male patients were current smokers when compared to female patients. Study Blondal2012 (30.3\% versus 7.00\%), study Buja2012 (52.1\% versus $16.5 \%)$, study Lin2013 (42.3\% versus $11.6 \%)$, and study Younan $2015(45.0 \%$ versus $3.00 \%)$ are a few examples that showed a higher percentage of smokers among men. This unexpected phenomenon called the smoking paradox could be another factor contributing to such a result $[18,19]$.

Recent research has also shown women with T2DM to be at higher risk of adverse events due to the onset mechanism of acute MI, the high burden of cardiac risk factors and conditions which are associated with the 


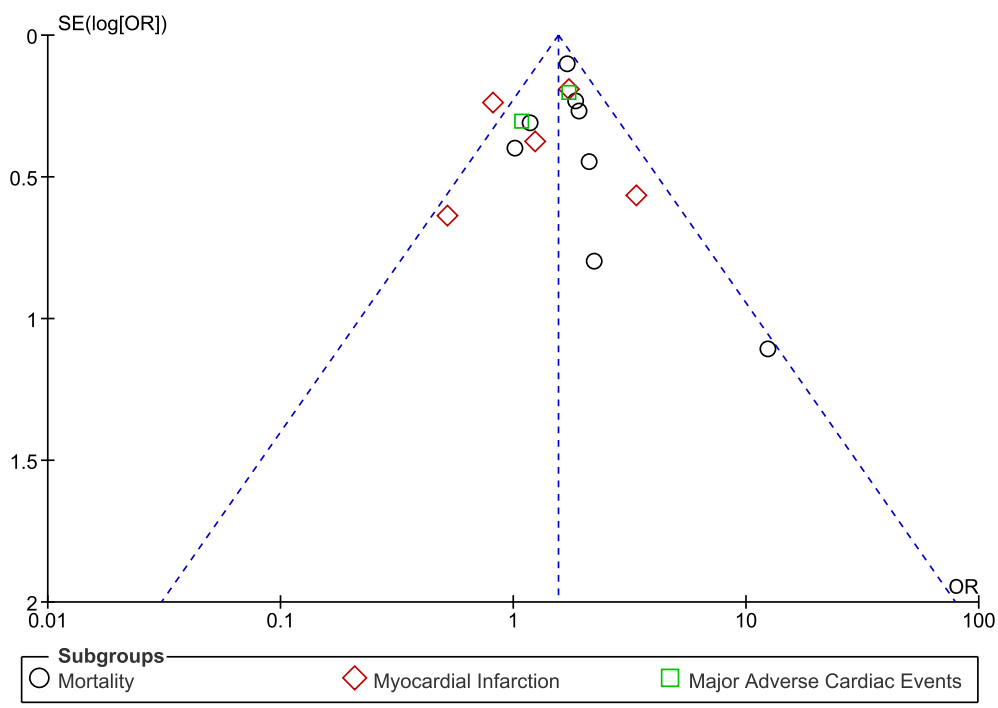

Fig. 12 Funnel plot representing publication bias across studies which assessed the short-term outcomes following percutaneous coronary intervention between male and female patients with diabetes mellitus

procedural success rate following $\mathrm{PCI}$ such as bleeding events [20,21]. Other studies have also shown women to be at a higher risk of developing diffuse small vessel disease which increased their chances of suffering from diabetic cardiomyopathy. In addition, some important biological differences were observed in the content of atherosclerotic plaque in men and women making women to have a more complicated disease or lesion compared to men with the same condition [22].
Also, the decrease or absence of ovarian hormones in menopause women, might further contribute to cardiovascular complications following PCI [23]. Moreover, endothelial dysfunction, functional contraction of the myocardium, platelet aggregations and other regulatory mechanisms might contribute to higher adverse cardiac events in women compared to men with the same clinical conditions [24, 25]. However, other factors should further be investigated.

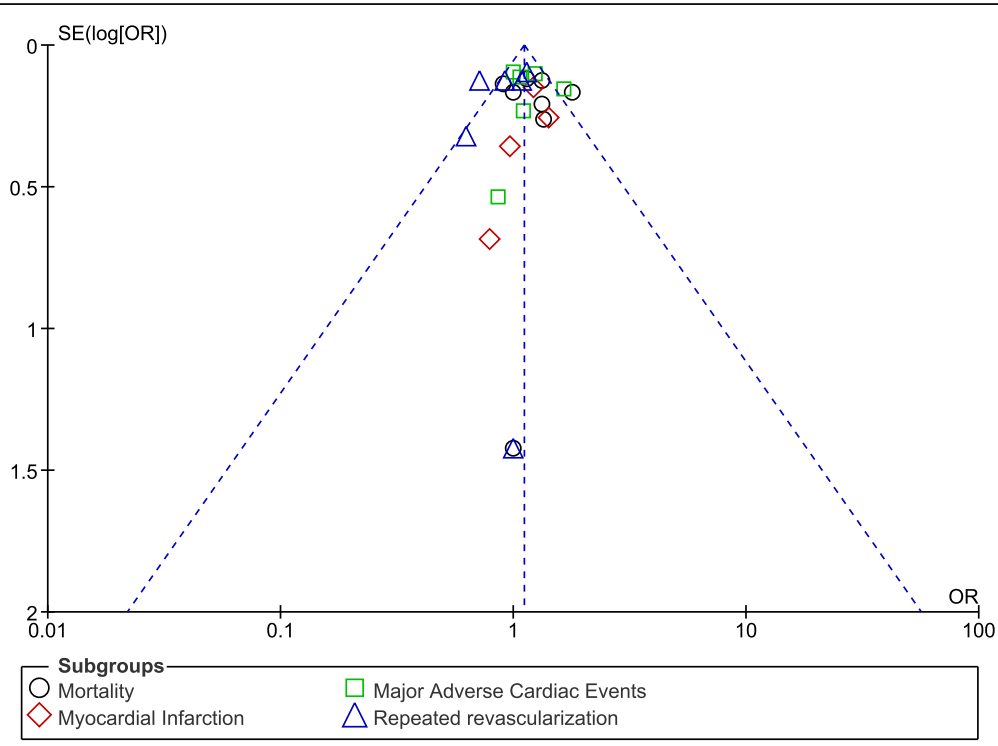

Fig. 13 Funnel plot representing publication bias across studies which assessed the long-term outcomes following percutaneous coronary intervention between male and female patients with diabetes mellitus 


\section{Novelty}

This research is new because:

- It is among the first few meta-analyses comparing adverse cardiovascular outcomes in male versus female patients with T2DM following PCI.

- This research represents an interesting idea which should be debated in clinical medicine.

- A very low or moderate level of heterogeneity was observed among several of the subgroups analyzing specific outcomes.

- A large number of patients from different corners of the globe were included compared to previously published articles.

\section{Limitations}

Limitations are as follows:

- A limited sample size could affect the results.

- Mainly data which were obtained from observational studies were included in this analysis.

- The endpoints were analyzed using a limited number of studies.

- Different follow up periods in different studies could have affected the results.

- The duration of diabetes mellitus, the length of dual anti-platelet therapy use and the use of other cardiovascular and oral anti hypoglycemic medications as well as the use of insulin therapy could also have had an effect on the outcomes between male and female patients with diabetes mellitus. Unfortunately, the medication profile was limitedly reported in these studies.

\section{Conclusions}

Following PCI, women with T2DM were indeed more susceptible to short and long-term cardiovascular complications compared to male patients with the same chronic disease. Even though this result was more applicable to patients with acute myocardial infarction, the fact that women were older with higher co-morbidities at baseline compared to men, should also not be ignored.

\section{Abbreviations}

MACEs: major adverse cardiac events; Ml: myocardial infarction;

$\mathrm{PCl}$ : percutaneous coronary intervention; T2DM: type 2 diabetes mellitus

\section{Acknowledgements}

Not applicable.

\section{Funding}

This research was supported by Youth Science Foundation of Guangxi Medical University (No. GXMUYSF201308), Scientific Project of Guangxi Higher Education (No. KY2015ZD028) and National Natural Science Foundation of China (No. 81560046).
Availability of data and materials

All data and materials used in this research are freely available in Medline/ PubMed. References have been provided.

\section{Authors' contributions}

$\mathrm{PKB}, \mathrm{MP}$ and $\mathrm{FH}$ were responsible for the conception and design, acquisition of data, analysis and interpretation of data, drafting the initial manuscript and revising it critically for important intellectual content. PKB wrote this manuscript. All authors read and approved the final manuscript.

Ethics approval and consent to participate

Ethical approval was not applicable for this systematic review and meta-analysis.

Consent for publication

Not applicable.

Competing interests

The authors declare that they have no competing interests.

\section{Publisher's Note}

Springer Nature remains neutral with regard to jurisdictional claims in published maps and institutional affiliations.

\section{Author details}

${ }^{1}$ Institute of Cardiovascular Diseases, the First Affiliated Hospital of Guangxi Medical University, Nanning, Guangxi 530027, People's Republic of China.

${ }^{2}$ Guangxi Medical University, Nanning, Guangxi 530027, People's Republic of China.

Received: 2 May 2017 Accepted: 24 July 2017

Published online: 27 July 2017

\section{References}

1. Wild S, Roglic G, Green A, Sicree R, King H. Global prevalence of diabetes: estimates for the year 2000 and projections for 2030. Diabetes Care. 2004; 27:1047-53.

2. Li N, Yang YG, Chen MH. Comparing the adverse clinical outcomes in patients with non-insulin treated type 2 diabetes mellitus and patients without type 2 diabetes mellitus following percutaneous coronary intervention: a systematic review and meta-analysis. BMC Cardiovasc Disord. 2016;16(1):238.

3. Naito R, Miyauchi K, Konishi H, Tsuboi S, Ogita M, Dohi T, Kasai T, Tamura H, Okazaki S, Isoda K, Daida H. Gender difference in long-term clinical outcomes following percutaneous coronary intervention during 1984-2008. Atherosclerosis. 2016:247:105-10.

4. de Boer SP, Roos-Hesselink JW, van Leeuwen MA, Lenzen MJ, van Geuns RJ, Regar E, van Mieghem NM, van Domburg R, Zijlstra F, Serruys PW, Boersma E. Excess mortality in women compared to men after PCI in STEMI: an analysis of 11,931 patients during 2000-2009. Int J Cardiol. 2014;176(2): 456-63.

5. Sotomi $Y$, Onuma $Y$, Cavalcante R, et al. Geographical Difference of the Interaction of Sex With Treatment Strategy in Patients With Multivessel Disease and Left Main Disease: A Meta-Analysis From SYNTAX (Synergy Between PCI With Taxus and Cardiac Surgery), PRECOMBAT (Bypass Surgery Versus Angioplasty Using Sirolimus-Eluting Stent in Patients With Left Main Coronary Artery Disease), and BEST (Bypass Surgery and Everolimus-Eluting Stent Implantation in the Treatment of Patients With Multivessel Coronary Artery Disease) Randomized Controlled Trials. Circ Cardiovasc Interv. 2017; 10(5):e005027.

6. Liberati A, Altman DG, Tetzlaff J, et al. The PRISMA statement for reporting systematic reviews and meta-analyses of studies that evaluate healthcareinterventions: explanation and elaboration. BMJ. 2009:339:b2700.

7. Higgins JP, Thompson SG, Deeks JJ, et al. Measuring inconsistency in metaanalyses. BMJ. 2003;327:557-60

8. Higgins JPT, Thompson SG, Deeks JJ, Altman DG. Measuring inconsistency in meta-analyses. BMJ. 2003;327(7414):557-60

9. Blöndal M, Ainla T, Marandi T, Baburin A, Eha J. Sex-specific outcomes of diabetic patients with acute myocardial infarction who have undergonepercutaneous coronary intervention: a register linkage study. Cardiovasc Diabetol. 2012;11:96. 
10. Roffi M, Radovanovic D, Erne P, Urban P, Windecker S, Eberli FR. AMIS plus investigator. Gender-related mortality trends among diabetic patients with ST-segment elevation myocardialinfarction: insights from a nationwide registry 1997-2010. Eur Heart J Acute Cardiovasc Care. 2013;2(4):342-9.

11. Liu HL, Liu Y, Hao ZX, Geng GY, Zhang ZF, Jing SB, Ba N, Guo W. Comparison of primary coronary percutaneous coronary intervention between diabetic men and women with acute myocardial infarction. Pak J Med Sci. 2015;31(2):420-5.

12. Tamis-Holland JE, Lu J, Korytkowski M, Magee M, Rogers WJ, Lopes N, Mighton L, Jacobs AK. BARI 2D study group. Sex differences in presentation and outcome among patients with type 2 diabetes and coronary artery disease treated with contemporary medical therapy with or without prompt revascularization: a report from the BARI 2D trial (bypass angioplasty revascularization investigation 2 diabetes). J Am Coll Cardiol. 2013;61(17): 1767-76.

13. Baber U, Giustino G, Sartori S, et al. Effect of chronic kidney disease in women undergoing percutaneous coronary intervention with drug-eluting stents: a patient-level pooled analysis of randomized controlled trials. JACC Cardiovasc Interv. 2016;9(1):28-38.

14. Kautzky-Willer A, Kamyar MR, Gerhat D, Handisurya A, Stemer G, Hudson S, Luger A, Lemmens-Gruber R. Sex-specific differences in metabolic control, cardiovascular risk, and interventions in patients with type2 diabetes mellitus. Gend Med. 2010:7(6):571-83.

15. Champney KP, Veledar E, Klein M, Samady H, Anderson D, Parashar S, Wenger N, Vaccarino V. Sex-specific effects of diabetes on adverse outcomes after percutaneous coronary intervention: trends over time. Am Heart J. 2007;153(6):970-8.

16. Conrotto F, D'Ascenzo F, Humphries KH, Webb JG, Scacciatella P, Grasso C, D'Amico M, Biondi-Zoccai G, Gaita F, Marra S. A meta-analysis of sex-related differences in outcomes after primary percutaneous intervention for STsegment elevation myocardial infarction. J Interv Cardiol. 2015;28(2):132-40.

17. Bundhun PK, Wu ZJ, Chen MH. Impact of modifiable cardiovascular risk factors on mortality after percutaneous coronary intervention: a systematic review and meta-analysis of 100 studies. Medicine (Baltimore). 2015;94(50):e2313.

18. Albertal M, Cura F, Escudero AG, Thierer J, Trivi M, Padilla LT, Belardi J, PREMIAR investigators. Mechanism involved in the paradoxical effects of active smoking following primary angioplasty: a subanalysis of the protection of distal embolization in high-risk patients with acute myocardial infarction trial. J Cardiovasc Med (Hagerstown). 2008;9(8):810-2.

19. Weisz G, Cox DA, Garcia E, Tcheng JE, Griffin JJ, Guagliumi G, Stuckey TD, Rutherford BD, Mehran R, Aymong E, Lansky A, Grines CL, Stone GW. Impact of smoking status on outcomes of primary coronary intervention for acute myocardial infarction-the smoker's paradox revisited. Am Heart J. 2005; 150(2):358-64

20. Lee C, Joseph L, Colosimo A, Dasgupta K. Mortality in diabetes compared with previous cardiovascular disease: a gender-specific meta-analysis. Diabetes Metab. 2012;38(5):420-7

21. Steinberg HO, Paradisi G, Cronin J, Crowde K, Hempfling A, Hook G, Baron AD. Type II diabetes abrogates sex differences in endothelial function in premenopausal women. Circulation. 2000;101(17):2040-6.

22. Mortensen $\mathrm{KH}$, Thuesen L, Kristensen IB, Christiansen EH. Spontaneous coronary artery dissection: a western Denmark heart registry study. Catheter Cardiovasc Interv. 2009;74(5):710-7

23. Vaccarino V, Badimon L, Corti R, de Wit C, Dorobantu M, Hall A, Koller A, Marzilli $M$, Pries A, Bugiardini R. Working group on coronary Pathophysiologyand microcirculation. Ischaemic heart disease in women: are there sex differences in pathophysiology and risk factors? Position paper from the working group on coronary pathophysiology and microcirculation of the European society of cardiology. Cardiovasc Res. 2011;90(1):9-17.

24. Jacobs AK. Coronary intervention in 2009: are women no different than men? Circ Cardiovasc Interv. 2009;2(1):69-78.

25. Graham MM, Ghali WA, Faris PD, Galbraith PD, Norris CM, Knudtson ML. APPROACH Investigators. Sex differences in the prognostic importance of diabetes in patients with ischemic heart disease undergoing coronary angiography. Diabetes Care. 2003;26(11):3142-7.

26. Buja P, D'Amico G, Facchin M, Barioli A, Napodano M, Capodanno D, Musumeci G, Frigo AC, Saia F, Menozzi A, De Benedictis M, Lee MS, Lettieri C, Tamburino C, Sardella G, Isabella G, Tarantini G. Gender-related differences of diabetic patients undergoing percutaneous coronary intervention with drug-eluting stents: a real-life multicenter experience. Int J Cardiol. 2013;168(1):139-43.
27. Lin GM, Li YH, Lin CL, Wang JH, Han CL. Gender differences in the impact of diabetes on mortality in patients with established coronary arterydisease: a report from the eastern Taiwan integrated health care delivery system of coronary heart disease(ET-CHD) registry, 1997-2006. J Cardiol. 2013;61(6):393-8.

28. Younan H, Ragab T, El-Khashab K, Farag N. Impact of gender difference on $\mathrm{PCl}$ outcome in Egyptian diabetic patients: prospective two center registry study. Egypt Heart J. 2015;67(1):55-61. http://dx.doi.org/10.1016/j.ehj.2014. 09.001.

29. Epps KC, Holper EM, Selzer F, Vlachos HA, Gualano SK, Abbott JD, Jacobs AK, Marroquin OC, Naidu SS, Groeneveld PW, Wilensky RL. Sex differences in outcomes following percutaneous coronary intervention according to age. Circ Cardiovasc Qual Outcomes. 2016;9(2 Suppl 1):S16-25.

30. Toyota T, Furukawa Y, Ehara N, Funakoshi S, Morimoto T, Kaji S, Nakagawa Y Kadota K, Iwabuchi M, Shiomi H, Yamamuro A, Kinoshita M, Kitai T, Kim K, Tani T, Kobori A, Kita T, Sakata R, Kimura T. Credo-Kyoto Investigators. Sexbased differences in clinical practice and outcomes for Japanese patients with acute myocardialinfarction undergoing primary percutaneous coronary intervention. Circ J. 2013;77(6):1508-17.

31. Cheng $\mathrm{Cl}$, Yeh $\mathrm{KH}$, Chang HW, Yu TH, Chen YH, Chai HT, Yip HK. Comparison of baseline characteristics, clinical features, angiographic results, and early outcomes in men vs women with acute myocardial infarction undergoing primary coronary intervention. Chest. 2004;126(1):47-53.

32. Eitel I, Desch S, de Waha S, Fuernau G, Gutberlet M, Schuler G, Thiele H. Sex differences in myocardial salvage and clinical outcome in patients with acute reperfused ST-elevation myocardial infarction: advances in cardiovascular imaging. Circ Cardiovasc Imaging. 2012;5(1):119-26.

33. Kosuge M, Kimura K, Kojima S, Sakamoto T, Ishihara M, Asada Y, Tei C, Miyazaki S, Sonoda M, Tsuchihashi K, Yamagishi M, Ikeda Y, Shirai M, Hiraoka $H$, Inoue $T$, Saito $F$, Ogawa $H$. Japanese acute coronary syndrome study (JACSS) investigators. Sex differences in early mortality of patients undergoing primary stenting for acute myocardial infarction. Circ J. 2006; 70(3):217-21.

34. Zhang Q, Qiu JP, Zhang RY, Li YG, He B, Jin HG, Zhang JF, Wang XL, Jiang L, Liao ML, Hu J, Shen WF. Absence of gender disparity in short-term clinical outcomes in patients with acute ST-segment elevationmyocardial infarction undergoing sirolimus-eluting stent based primary coronary intervention: a report from shanghai acute coronary event (SACE) registry. Chin Med J. 2010;123(7):782-8

35. Zanchi J, Mirić D, Giunio L, Vuković I, Marković B, Duplancić D, Kristić I. Gender differences in in-hospital mortality and angiographic findings of patients with acute ST-segment elevation myocardial infarction (STEMI) undergoing percutaneous coronary intervention (PCI). Coll Antropol. 2009; 33(4):1359-62.

\section{Submit your next manuscript to BioMed Central and we will help you at every step:}

- We accept pre-submission inquiries

- Our selector tool helps you to find the most relevant journal

- We provide round the clock customer support

- Convenient online submission

- Thorough peer review

- Inclusion in PubMed and all major indexing services

- Maximum visibility for your research

Submit your manuscript at www.biomedcentral.com/submit
) Biomed Central 